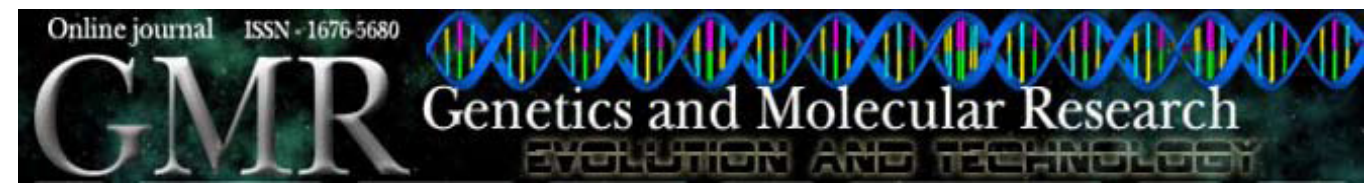

\title{
The Caenorhabditis elegans genome: a multifractal analysis
}

\author{
P.E. Vélez ${ }^{1}$, L.E. Garreta ${ }^{2}$, E. Martínez ${ }^{3}$, N. Díaz ${ }^{3}$, S. Amador ${ }^{3}$, I. Tischer ${ }^{2}$, \\ J.M. Gutiérrez ${ }^{4}$ and P.A. Moreno ${ }^{2}$ \\ ${ }^{1}$ Departamento de Biología, Universidad del Cauca, Popayán, Colombia \\ ${ }^{2}$ Escuela de Ingeniería de Sistemas y Computación, \\ Universidad del Valle, Santiago de Cali, Colombia \\ ${ }^{3}$ Escuela de Ingeniería de Sistemas y Computación, \\ Universidad del Cauca, Popayán, Colombia \\ ${ }^{4}$ Instituto de Física de Cantabria, \\ Universidad de Cantabria-CSIC, Santander, Spain \\ Corresponding author: P.A. Moreno \\ E-mail: pedro.moreno@correounivalle.edu.co
}

Genet. Mol. Res. 9 (2): 949-965 (2010)

Received January 10, 2010

Accepted February 25, 2010

Published May 25, 2010

DOI 10.4238/vol9-2gmr756

\begin{abstract}
The Caenorhabditis elegans genome has several regular and irregular characteristics in its nucleotide composition; these are observed within and between chromosomes. To study these particularities, we carried out a multifractal analysis, which requires a large number of exponents to characterize scaling properties. We looked for a relationship between the genetic information content of the chromosomes and multifractal parameters and found less multifractality compared to the human genome. Differences in multifractality among chromosomes and in regions of chromosomes, and two group averages of chromosome regions were observed. All these differences were mainly dependent on differences in the contents of repetitive DNA. Based on these properties, we propose a nonlinear model for the structure of the $C$. elegans genome, with some biological implications. These results suggest that examining
\end{abstract}


differences in multifractality is a viable approach for measuring local variations of genomic information contents along chromosomes. This approach could be extended to other genomes in order to characterize structural and functional regions of chromosomes.

Key words: Caenorhabditis elegans genome; Multifractal analysis; Repetitive DNA sequences; Chaos game representation

\section{INTRODUCTION}

Several tens of Eukaryotic genomes have already been sequenced. These genomes have revealed a complex structure (Lewin, 2008) that is highly regionalized, which can be studied by methods that allow measuring how the information content is fragmented.

Information theory has been a very useful conceptual framework to study the information content along a symbol (or signal) sequence. Its beginnings can be traced to discussions about entropy of information. These concepts were rigorously formulated in other terms by Shannon (1948) in the theory of information for communication. During the 60's Mandelbrot (1982) established fractal geometry, as a new geometry able to measure the irregularity of nature. The fractal geometry paradigm introduces several ways to measure this information content by calculating the fractal dimension, an exponent derived from a power law, which gives us an idea of the fragmentation level or information content of a complex phenomenon. Fractal geometry is a useful approach for searching for self-similar properties in biological structures and processes (Goldberger, 1996). These self-similarities are organized following scaling or power laws, which emerge from the spatial-temporal, extended interaction of the different system components (Voss, 1992). These systems have a complex structure, which result from deterministic as well as random events, leading to an apparently disordered distribution. Thus, power laws are powerful tools to quantify the scaling properties of information contents.

Fractal geometry has been a useful approach for addressing several problems related to coding and non-coding DNA sequences, phylogenetic relationships, and for searching for explanations of regularities observed in molecular data bases. These studies were initially focused on long-range correlation properties in DNA sequences. Other surveys applying power laws have been reported (for a detailed review, see Yu et al., 2001).

Sometimes, one exponent may not be enough to characterize a complex phenomenon; so that more exponents are required. Multifractal formalism allows for this. It is applied when many fractal subsets with different scaling properties (with a large number of exponents or fractal dimensions) coexist simultaneously. As a result, when a spectrum of singularities of multifractal measure is generated, the scaling behavior of the symbol frequencies of a sequence can be quantified.

Multifractal analysis has been implemented to improve characterization of spatial inhomogeneity of both theoretical and experimental fractal patterns (Hentschel and Procaccia, 1983; Peitgen et al., 1992) in physics, chemistry (Stanley and Meakin, 1988), economy, and ecology. For example, it has been applied to study the turbulence phenomenon, time series analysis, and financial modeling. It has also been useful in studying different kinds of problems at DNA and protein sequence levels, under two modalities: 1) by using subintervals in a 
one-dimensional (1-D) space to represent substrings and 2) by using a 2-D space in the chaos game representation context (Jeffrey, 1990). For a detailed review using the chaos game representation, see Yu et al. (2004).

The first modality was applied in pre-genomics times, to study DNA sequences by making spectral and multifractal analyses of measurements (Berthelsen et al., 1994). It was then used to reconstruct phylogeny from mitochondrial DNA (Glazier et al., 1995). Subsequently, in post-genomics times, multifractal analysis was used in discriminate analyses of complete bacterial genomes (Yu et al., 2001) and to distinguish coding and non-coding sequences in DNA sequences (Zhou et al., 2005). It has been useful in bacterial analysis to construct phylogenetic trees (Yu et al., 2003) and clustering of protein structures due to hydrophobic free energy and solvent accessibility of proteins (Yu et al., 2006). Recently, multifractal analysis has shown its utility in predicting the existence of promoters for RNA polymerase II (Yang et al., 2008).

In the second modality, multifractality analysis applied to chaos game representation patterns was first used on DNA discrete sequences (Gutiérrez et al., 1998); this was extended to a genomic level in a preliminary study (Moreno et al., 2000). Any influence of coding and non-coding content was observed in the correlation length of a given sequence (Gutiérrez et al., 2001). Bacteria classification based on the detailed Hydrophobic-Polar model in proteins revealed the utility of this approach (Yu et al., 2004).

In short, most of these approaches have practical applications for classifying microorganisms, distinguishing coding and non-coding sequences, studying proteins, and for promoter prediction. Much less attention has been paid to their use to characterize structural genetics information content. To apply multifractal formalism to this problem, we chose the Caenorhabditis elegans genome.

For a long time, C. elegans has been an excellent model for various types of studies (Wood, 1987; Brenner and Miller, 2002). Its genome was the first sequenced eukaryotic genome. Its molecular structure is particularly homogenous along the chromosome sequences (Anonymous, 1998), due to the presence of several regular characteristics. For example, with an average size of $3000 \mathrm{bp}$, genes are usually small and close to $50 \%$ of introns are extremely small, being about $47 \mathrm{bp}$ long (Spieth and Lawson, 2006). Also, several bioinformatics studies have identified a remarkable periodicity for AA/TT dinucleotides, with periodicity at each $10 \mathrm{bp}$ (hyperperiodicity; Moreno-Herrero et al., 2006); DNA periodicity in exons is determined by codon usage frequencies (Eskesen et al., 2004). All these characteristics contribute to producing homogenous nucleotide topography along the $C$. elegans genome.

Sequencing of the C. elegans genome revealed a regional organization of chromosomes, given by a distinctive distribution of some repeats and highly conserved genes (Anonymous, 1998). That is, the C. elegans genome also has some irregular characteristics. For example, gene density is a little higher in the central regions of the chromosomes than in the chromosome arms, and the vast majority of several kinds of DNA repeats are located in the chromosome arms rather than in the central regions (Figure 1, Panel A1). Additionally, mutation (Wolfe et al., 1989) and recombination (Wood, 1987) rates are more frequent in the chromosome arms, and (G+C) contents are slightly higher in the chromosome arms than in their central regions.

Given these regular and irregular characteristics, we decided to determine if some of these distributions can be analyzed by a multifractal formalism. 


\section{MATERIAL AND METHODS}

\section{Data bases, sequences, and multifractal approaches}

Chromosome 21 from Homo sapiens [hs_ref chr21 build 36.1, contig NT_011512.10 (from 28.6 Mbp we took $\sim 15 \mathrm{Mbp}$ )] and C. elegans $\mathrm{NC}$ _003279-84.fna, data bases were downloaded from the NCBI web site. Four approaches were used to measure multifractality in the C. elegans genome. 1) In comparison with the human chromosome. The multifractal spectra for chromosome 21 of H. sapiens and chromosome I of C. elegans were studied. 2) By chromosome. Here the DNA sequences of five allosomes (I-V) and one sexual chromosome (X) were analyzed. 3) By chromosome region. We took a straightforward approach: the complete length of each chromosome was divided by 3 . These regions were called L (left), C (center), and R (right), and the resulting 18 DNA sequences were analyzed by multifractal analysis (See Figure 1, Panel A1). 4) By group averages. Here the molecular and multifractal parameters for $6 \mathrm{C}$ regions of the C. elegans chromosomes were averaged and compared with the averages of the $12 \mathrm{~L}$ and $\mathrm{R}$ regions of the chromosomes.

\section{Molecular parameters: contents of $(\mathrm{G}+\mathrm{C})$, DNA repeats, and genes}

Using scripts in Python, the $(\mathrm{G}+\mathrm{C})$ and repeat TTAGGC contents and gene density were calculated for each chromosome and fragment of chromosomes of C. elegans. Likewise, the dinucleotide AA/TT contents were counted for each DNA fragment for the motifs: $\mathrm{AAN}_{10} \mathrm{AA}$ and TTN ${ }_{10} \mathrm{TT}$. Additionally, other DNA repeat (AAAAT, AAATT, TTTTC, and TTTTT) contents were examined in each chromosome fragment.

\section{Chaos game representation of DNA sequences}

According to Jeffrey (1990) and Yu et al. (2004), the chaos game representation for a given DNA sequence $s=s_{1}, \ldots s_{N}$ is obtained as follows: the corners of the square $[0,1] \times[0$, 1] are labeled with A, C, G, and T. The first point of $s$ is plotted halfway between the center of the square and the corner corresponding to the first nucleotide of $s$; the $i$-th point of $s$ is then plotted halfway between the $(i-1)$-th point and the corner corresponding to the $i$-th nucleotide. An example of a chaos game representation is shown in Figure 1, Panel A2.

\section{Multifractal analysis and discrimination analysis}

In spite of arguments for the sandbox method (Tél et al., 1989; Yu et al., 2004), we decided to use the box-counting method for our multifractal spectrum estimation, because it counts boxes in a straightforward manner and maintains statistics allowing dimension calculation (Peitgen et al., 1992). Given a grid of mesh size $\varepsilon$ over the fractal, the number $M_{i}$ of fractal points that fall in the $i$-th grid box is determined and related to the total number $M_{0}$. The multifractal spectrum $D_{q}^{b c}$ based on the box-counting method (Mandelbrot, 1982; Halsey et al., 1986) is defined as:

$$
D_{q}(\varepsilon)=\frac{\ln \left(\sum_{i}\left(M_{i} / M_{0}\right)^{q}\right)}{\ln (\varepsilon)} \frac{1}{q-1},
$$

(Equation 1) 
where $q$ is an integer parameter. The multifractal dimension is defined as

$$
D_{q}=\lim _{\varepsilon \rightarrow 0} D_{q}^{b c}(\varepsilon)
$$

(Equation 2)

A practical use of the box-counting method transforms Equation 1 into

$$
\ln \left(M_{i}^{q}\right)=D_{q}^{b c}(\varepsilon)(q-1) \ln (\varepsilon)+(q-1) \ln \left(M_{0}^{q}\right) \quad \text { (Equation 3) }
$$

and performs a linear fit of $\ln \left(M_{i}^{q}\right) v s(q-1) \ln (\varepsilon)$, using sample data of $M_{i}$. The slope that is obtained is an approximation of the multifractal dimension $\mathrm{D}_{q}$. From Equation 3 it can be inferred that a high $\mathrm{D}_{q}$ for positive $q$ values emphasizes regions where the points are dense; for negative $q$ values, it is associated with the structure and properties of sparse regions. Directly from the multifractal dimension $\mathrm{D}_{q}$, the correlation exponent $\tau(q)$ is derived as $\tau(q)=(q-1) \mathrm{D}_{q}$, which will also be used in some of our analyses. The degree of multifractality, $\Delta \mathrm{D}_{q}$, is defined as the difference between maximum and minimum values of $\mathrm{D}_{q}: \Delta \mathrm{D}_{q}=\mathrm{D}_{q \max }-\mathrm{D}_{q \min }$ (Dewey and Strait, 1996; Ivanov et al., 1999). If $\Delta \mathrm{D}_{q}$ is high, the multifractal spectrum is rich in information; for a small $\Delta \mathrm{D}_{q}$, the resulting dimension spectrum is poor in information.

In this study, the effects of the different DNA lengths on multifractality were not taken into account (for theory concerning this, see Fu et al. (2007)). Finally, the discrimination analyses were performed by using plots in 2-D and 3-D spaces, with combined molecular and multifractal parameters.

\section{RESULTS}

The box-counting method used here proved to be a good estimator for multifractal parameters in the C. elegans genome. It has been argued that the sandbox method is a more precise method than the box counting method (Yu et al., 2004). However, the box counting method is easy, has high computational efficiency and the program can be carried out for shapes with and without self-similarity (Peitgen et al., 1992), as those patterns shown for the chaos game representation. We used four approaches of multifractal analysis to examine the C. elegans genome.

\section{Multifractal comparison between the $C$. elegans chromosomes and human chromosomes}

The $C$. elegans multifractal spectra revealed marked multifractality reduction compared with human DNA sequences (see also Moreno et al., 2000). Here, we examined whether such a behavior is consistent at the chromosome level. Due to its intermediate repeat and gene densities, we selected human chromosome 21 as a landmark for comparison (Venter et al., 2001). Chromosome 21 is more multifractal ( 0.27 more) than the $C$. elegans chromosome I (Figure 1, Panels B1,B2; Table 1), suggesting that the genetics information along the C. elegans chromosome is more regularly ordered in comparison with the human genome. Human chromosome 21 is known to have a highly asymmetric complex structure that is aperiodic and rich in heterogeneous genetic information (Venter et al., 2001). 
A1.


A2. C

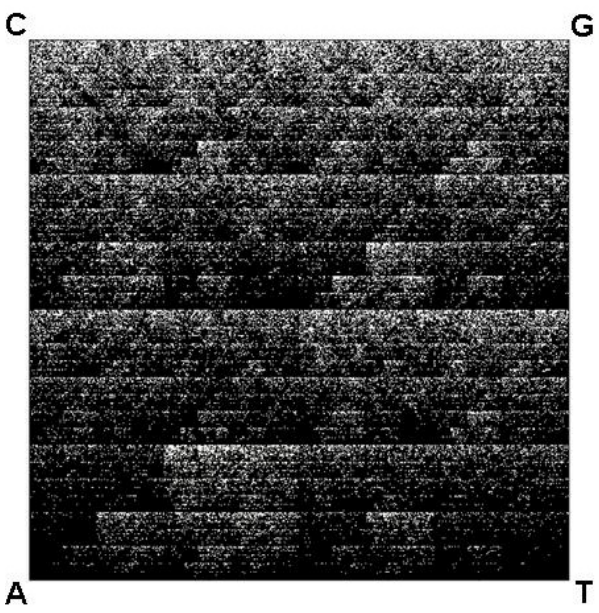

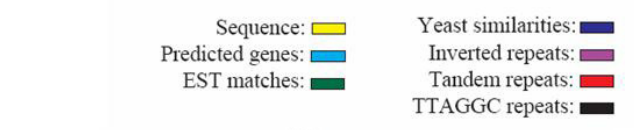
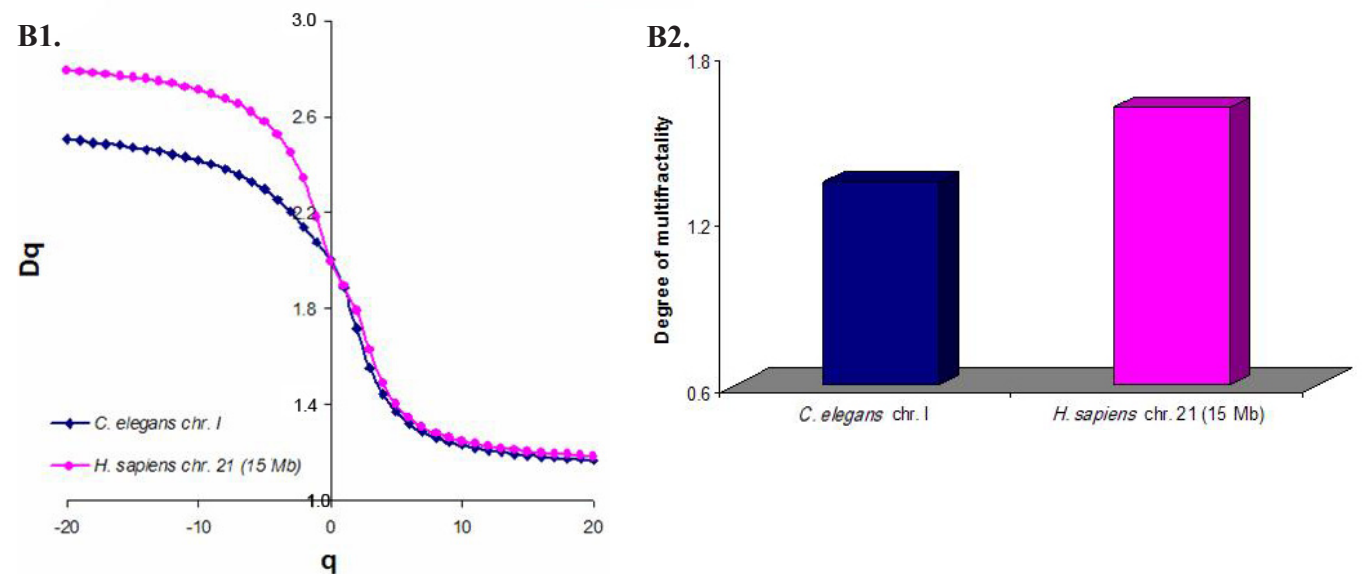

Figure 1. A1. Map of the Caenorhabditis elegans genome showing regular and irregular distributions in the contents of predicted genes and repetitive DNA (adapted from Figure 3 in Anonymous, 1998). The horizontal dashed lines show how each chromosome was divided into three regions ( $\mathrm{L}=$ left; $\mathrm{C}=$ center; $\mathrm{R}=$ right). $\mathbf{A 2}$. Chaos game representation of a C. elegans chromosome I fragment $(\sim 500 \mathrm{kbp})$. Note the high concentration of $(\mathrm{A}+\mathrm{T})$ content in the sub-quadrants AA and TT. Multifractal comparative analysis: B1. Comparison between the multifractal spectra for Caenorhabditis elegans and Homo sapiens. B2. Multifractality for both chromosomes.

Table 1. Multifractal parameters for two eukaryote chromosomes.

\begin{tabular}{lccccccc}
\hline & D-20 & D-2 & D-1 & D1 & D2 & D20 & $\Delta$ Dq \\
\hline C. elegans chr. I & 2.5044 & 2.1416 & 2.0773 & 1.8894 & 1.7143 & 1.1679 & 1.3365 \\
H. sapiens chr. 21 (15 Mb) & 2.7909 & 2.3422 & 2.1795 & 1.8947 & 1.7918 & 1.1843 & 1.6066 \\
\hline
\end{tabular}

$\mathrm{D} 1$ is called the information dimension and $\mathrm{D} 2$ the correlation dimension of the measurement. 


\section{Multifractal analysis by chromosome}

We studied the multifractal behavior of each C. elegans chromosome. We obtained the generalized dimension spectrum for each chromosome and used it to examine genetic information content (Figure 1, Panel A1). Chromosome III of C. elegans has high multifractality, whereas chromosome $\mathrm{X}$ has the lowest multifractality, suggesting strong periodicity of the nucleotides along the chromosome $\mathrm{X}$ sequence, and a high aperiodicity on chromosome III (Figure 2A,B; Table 2A). Discrimination analysis placed chromosomes X and III of C. elegans at opposite coordinates along the linear regression (Figure 2C). Likewise a low density of genes and repeats was found on chromosome $\mathrm{X}$ when compared with the other chromosomes (Table 2B).

$\mathbf{A}$

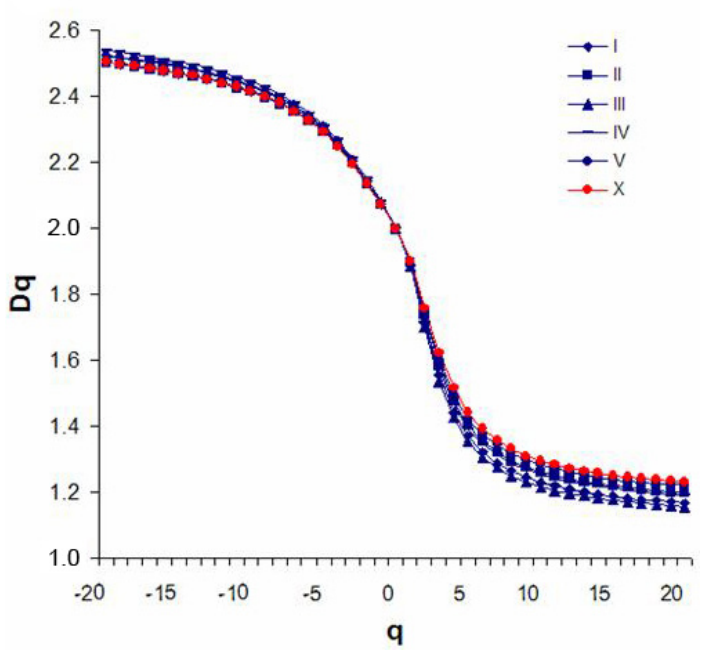

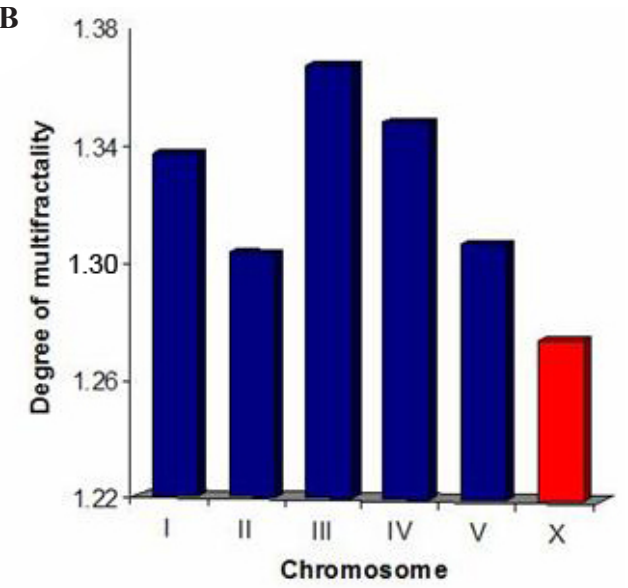

C

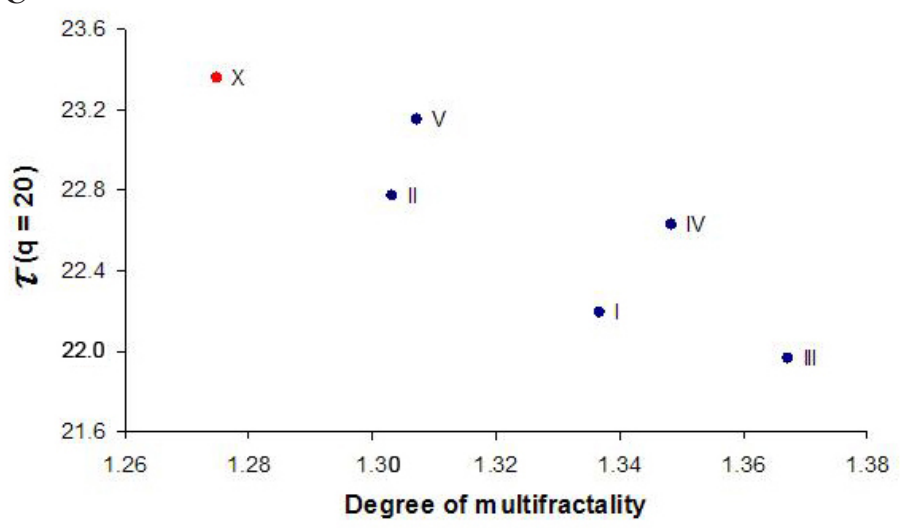

Figure 2. Multifractal analysis by chromosome. A. Dimension spectra for the Caenorhabditis elegans chromosomes. B. Degree of multifractality of Figure 2A. C. Discrimination method based on multifractal formalism in a distribution of two-dimensional points, $\mathrm{R}^{2} \sim 0.8$. 


\begin{tabular}{|c|c|c|c|c|c|c|c|c|}
\hline \multicolumn{9}{|c|}{ A. Multifractal parameters by chromosome. } \\
\hline Chr. & D-20 & D-2 & D-1 & D1 & D2 & D20 & $\Delta \mathrm{Dq}$ & $\tau(\mathrm{q}=20)$ \\
\hline I & 2.505 & 2.142 & 2.077 & 1.889 & 1.714 & 1.168 & 1.337 & 22.190 \\
\hline II & 2.502 & 2.137 & 2.073 & 1.899 & 1.74 & 1.198 & 1.303 & 22.770 \\
\hline III & 2.523 & 2.145 & 2.079 & 1.885 & 1.702 & 1.156 & 1.367 & 21.962 \\
\hline IV & 2.54 & 2.151 & 2.082 & 1.885 & 1.718 & 1.191 & 1.348 & 22.631 \\
\hline $\mathrm{V}$ & 2.526 & 2.14 & 2.075 & 1.897 & 1.743 & 1.218 & 1.307 & 23.149 \\
\hline $\mathrm{X}$ & 2.504 & 2.134 & 2.073 & 1.901 & 1.76 & 1.229 & 1.275 & 23.358 \\
\hline
\end{tabular}

B. Molecular parameters by chromosome.

\begin{tabular}{|c|c|c|c|c|c|c|}
\hline Chr. & Size $(\mathrm{Mb})$ & $(\mathrm{G}+\mathrm{C})$ & TTAGGC & Density (repeat/Mb) & Genes & Density (genes/Mb) \\
\hline I & 15.07 & 35.75 & 3742 & 248 & 3327 & 221 \\
\hline II & 15.28 & 36.2 & 3466 & 227 & 3968 & 260 \\
\hline III & 13.78 & 35.66 & 3195 & 232 & 3168 & 230 \\
\hline IV & 17.49 & 34.6 & 2890 & 165 & 3706 & 212 \\
\hline V & 20.92 & 35.43 & 3912 & 187 & 5392 & 258 \\
\hline $\mathrm{X}$ & 17.72 & 35.2 & 2271 & 128 & 3276 & 185 \\
\hline
\end{tabular}

\section{Multifractal analysis by chromosome region}

The most irregular characteristic exhibited by the C. elegans genome was examined. With the exception of chromosome $\mathrm{X}$, this asymmetric distribution is strongest on chromosomes I-V. Hence, we divided each chromosome into three fragments of DNA sequences (Figure 1, Panel A1). A detailed analysis for each generalized dimension spectrum revealed that the $\mathrm{C}$ regions of each chromosome have somewhat less multifractality than the multifractal spectra of the $\mathrm{L}$ and $\mathrm{R}$ regions (Figure $3 \mathrm{~A}$, Table $3 \mathrm{~A}$ ). The scaling exponent $\tau(q)$ can reveal other aspects of chromosome structure. Monofractal behavior would correspond to a straight line for $\tau(q)$; for multifractal behavior, $\tau(q)$ is nonlinear. The differences between the curves for chromosome arms and $\mathrm{C}$ regions can be seen in Figure 3B. The changing curvature for the data for the chromosome arms indicates multifractality. In contrast, $\tau(q)$ tends to be linear for the $\mathrm{C}$ regions of the chromosomes, indicating partial loss of multifractality.

We hypothesized that these two kinds of behaviors were due to different repetitive DNA sequence contents. Counts of different kinds of repetitive DNA sequences confirmed a clear contribution of these sequences in increasing the multifractality degree in the $\mathrm{L}$ and $\mathrm{R}$ regions of C. elegans chromosomes (Table 3B). A similar result can be observed by calculating the percentage in size of seven types of repeats present in each chromosome fragment (Figure 3C, Table 3C). The densities of DNA repeats were also found to be correlated with the multifractal degree (Table 3B; Supplementary material). Discrimination methods based on distributions of 2-D and 3-D points show how $\mathrm{C}$ regions can easily be discriminated from $\mathrm{L}$ and $\mathrm{R}$ region points (Figure 3D,E). Plots (D-1, D1, D-2) also allow discriminating C regions from $\mathrm{L}$ and $\mathrm{R}$ regions (Supplementary Material). 

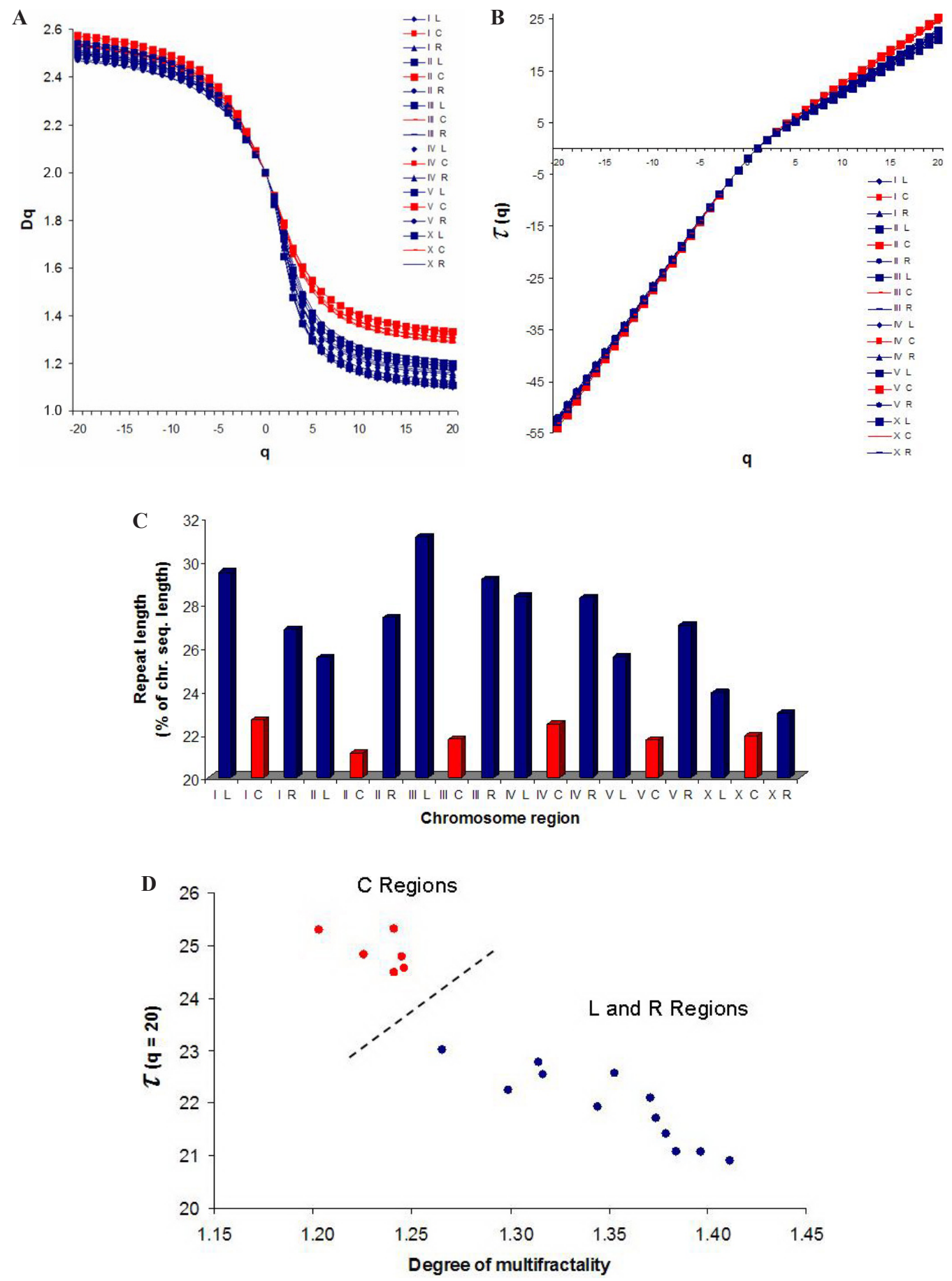

Figure 3. Continued on next page. 


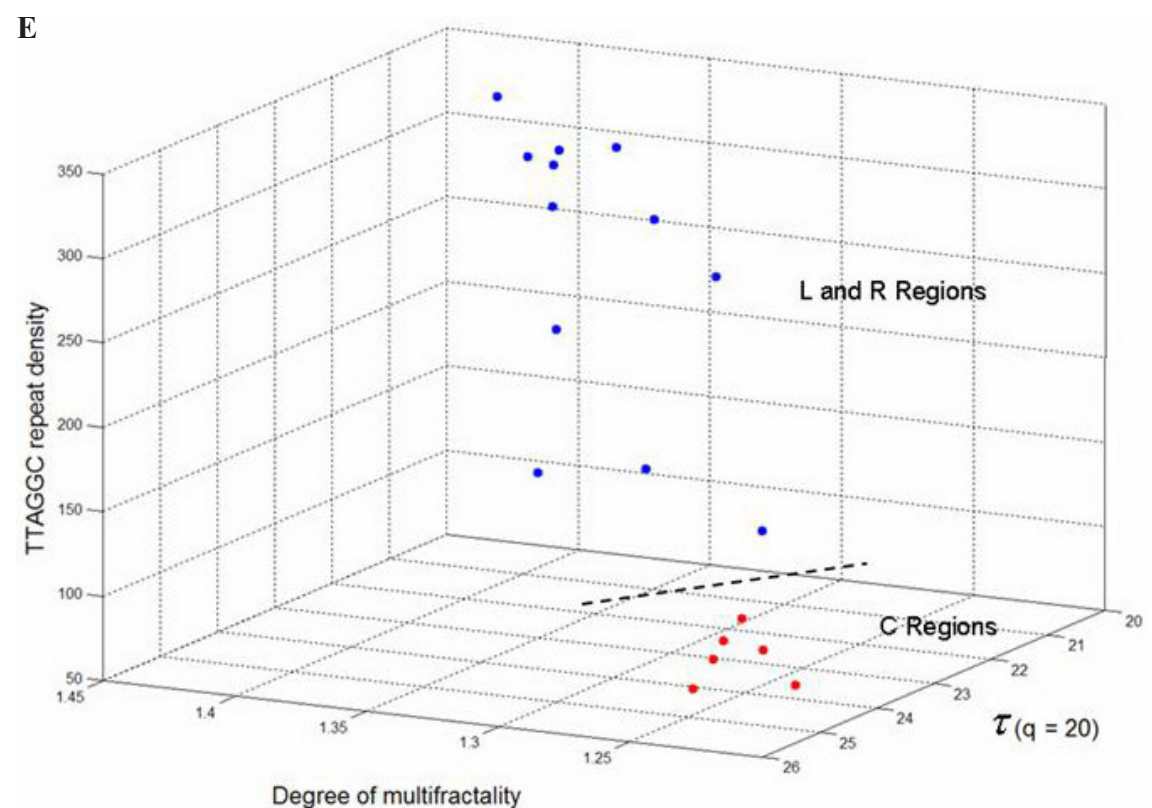

Figure 3. Multifractality by chromosome region. A. Generalized dimension spectrum for each chromosome region. B. Multifractal spectrum $\tau(q)$ for each chromosome region. C. Percentage of length for seven kinds of repeats. D. Discrimination method based on a distribution of two-dimensional points, $\mathrm{R}^{2}=0.914$. Blue points, $\mathrm{L}$ and $\mathrm{R}$ chromosome regions; red points, chromosome $\mathrm{C}$ regions. This multifractal approach discriminates $\mathrm{C}$ regions from $\mathrm{L}$ and $\mathrm{R}$ chromosome regions. E. Discrimination method based on two multifractal parameters and one molecular parameter. Each chromosome fragment dataset is characterized by three quantities. The first quantity (x-axis) is the degree of multifractality for each chromosomic region. The second quantity (y-axis) is the density of TTAGGC content of the chromosomic fragments. The third quantity (z-axis) is the exponent value $\tau(\mathrm{q}=20)$, characterizing the scaling of the 20th moment. $\mathrm{L}=$ left; $\mathrm{C}=$ center; $\mathrm{R}=$ right.

Table 3A. Multifractal parameters by chromosome region.

\begin{tabular}{lcccccccc}
\hline Chr. region & D-20 & D-2 & D-1 & D1 & D2 & D20 & $\Delta$ Dq & $\tau(\mathrm{q}=20)$ \\
\hline I L & 2.512 & 2.151 & 2.084 & 1.872 & 1.653 & 1.100 & 1.412 & 20.907 \\
I C & 2.539 & 2.170 & 2.090 & 1.895 & 1.766 & 1.293 & 1.246 & 24.564 \\
I R & 2.498 & 2.142 & 2.078 & 1.889 & 1.704 & 1.154 & 1.345 & 21.923 \\
II L & 2.503 & 2.138 & 2.074 & 1.898 & 1.728 & 1.186 & 1.317 & 22.541 \\
II C & 2.534 & 2.168 & 2.088 & 1.902 & 1.786 & 1.331 & 1.203 & 25.290 \\
II R & 2.506 & 2.140 & 2.077 & 1.887 & 1.686 & 1.126 & 1.379 & 21.403 \\
III L & 2.494 & 2.151 & 2.085 & 1.868 & 1.649 & 1.109 & 1.384 & 21.076 \\
III C & 2.533 & 2.163 & 2.086 & 1.902 & 1.776 & 1.307 & 1.226 & 24.828 \\
III R & 2.505 & 2.151 & 2.084 & 1.873 & 1.660 & 1.109 & 1.397 & 21.068 \\
IV L & 2.516 & 2.144 & 2.080 & 1.882 & 1.688 & 1.142 & 1.374 & 21.699 \\
IV C & 2.550 & 2.174 & 2.092 & 1.894 & 1.766 & 1.305 & 1.245 & 24.787 \\
IV R & 2.533 & 2.165 & 2.092 & 1.870 & 1.684 & 1.162 & 1.371 & 22.082 \\
V L & 2.540 & 2.144 & 2.077 & 1.894 & 1.723 & 1.187 & 1.353 & 22.553 \\
V C & 2.574 & 2.172 & 2.090 & 1.899 & 1.782 & 1.333 & 1.241 & 25.325 \\
V R & 2.469 & 2.141 & 2.077 & 1.890 & 1.708 & 1.170 & 1.299 & 22.239 \\
X L & 2.513 & 2.139 & 2.075 & 1.898 & 1.742 & 1.199 & 1.314 & 22.773 \\
X C & 2.529 & 2.152 & 2.081 & 1.901 & 1.774 & 1.288 & 1.241 & 24.474 \\
X R & 2.477 & 2.141 & 2.076 & 1.900 & 1.754 & 1.211 & 1.266 & 23.005 \\
\hline L & & & & & & &
\end{tabular}

$\mathrm{L}=$ left; $\mathrm{C}=$ center; $\mathrm{R}=$ right. 


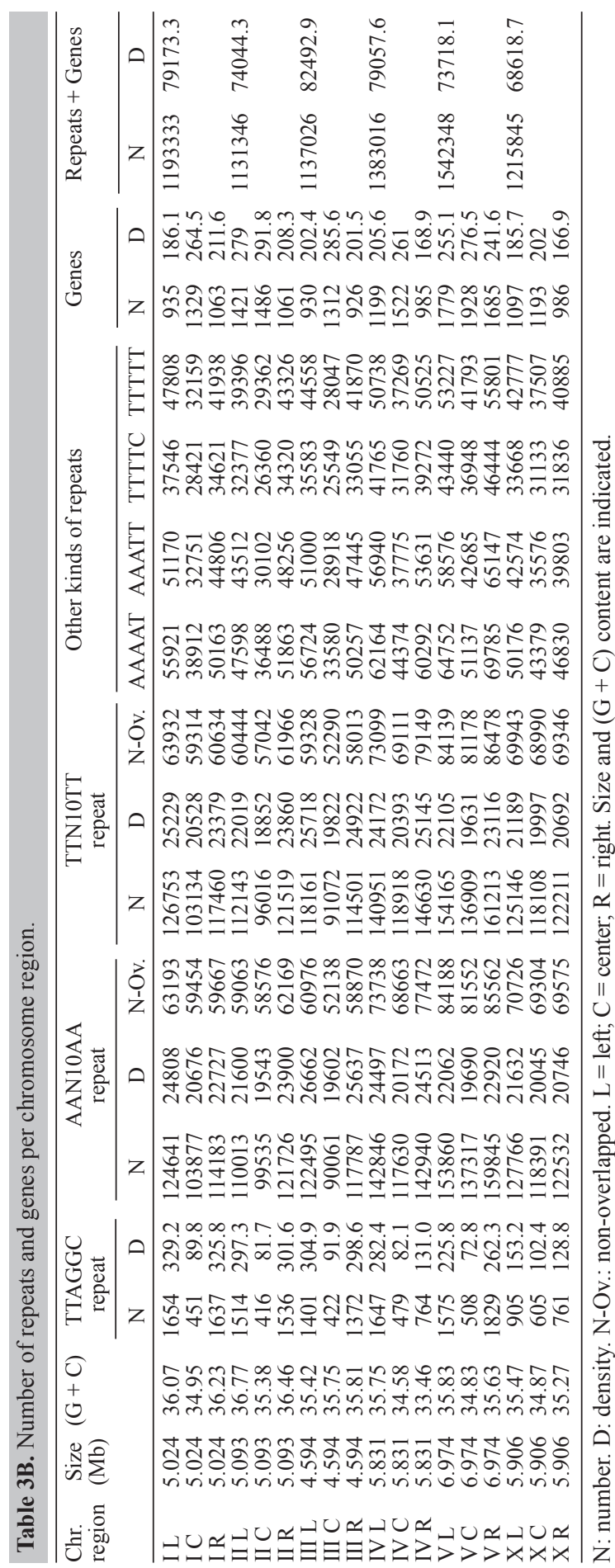




\begin{tabular}{|c|c|c|c|c|c|c|c|c|}
\hline Chr. region & TTAGGC & AAN10AA & TTN10TT & AAAAT & AAATT & TTTTC & TTTTT & Percent \\
\hline I L & 9924 & 252772 & 255728 & 279605 & 255850 & 187730 & 239040 & 29.47 \\
\hline I C & 2706 & 237816 & 237256 & 194560 & 163755 & 142105 & 160795 & 22.67 \\
\hline I R & 9822 & 238668 & 242536 & 250815 & 224030 & 173105 & 209690 & 26.84 \\
\hline II L & 9084 & 236252 & 241776 & 237990 & 217560 & 161885 & 196980 & 25.55 \\
\hline II C & 2496 & 234304 & 228168 & 182440 & 150510 & 131800 & 146810 & 21.14 \\
\hline II R & 9216 & 248676 & 247864 & 259315 & 241280 & 171600 & 216630 & 27.38 \\
\hline III L & 8406 & 243904 & 237312 & 283620 & 255000 & 177915 & 222790 & 31.10 \\
\hline III C & 2532 & 208552 & 209160 & 167900 & 144590 & 127745 & 140235 & 21.78 \\
\hline III R & 8232 & 235480 & 232052 & 251285 & 237225 & 165275 & 209350 & 29.14 \\
\hline IV L & 9882 & 294952 & 292396 & 310820 & 284700 & 208825 & 253690 & 28.39 \\
\hline IV C & 2874 & 274652 & 276444 & 221870 & 188875 & 158800 & 186345 & 22.46 \\
\hline IV R & 4584 & 309888 & 316596 & 301460 & 268155 & 196360 & 252625 & 28.29 \\
\hline V L & 9450 & 336752 & 336556 & 323760 & 292880 & 217200 & 266135 & 25.56 \\
\hline V C & 3048 & 326208 & 324712 & 255685 & 213425 & 184740 & 208965 & 21.75 \\
\hline V R & 10974 & 342248 & 345912 & 348925 & 325735 & 232220 & 279005 & 27.03 \\
\hline X L & 5430 & 282904 & 279772 & 250880 & 212870 & 168340 & 213885 & 23.94 \\
\hline $\mathrm{X} \mathrm{C}$ & 3630 & 277216 & 275960 & 216895 & 177880 & 155665 & 187535 & 21.92 \\
\hline X R & 4566 & 278300 & 277384 & 234150 & 199015 & 159180 & 204425 & 22.98 \\
\hline Average & & & & & & & & 25.41 \\
\hline
\end{tabular}

The percentages were calculated as follows: Percent $=\sum$ (for 7 types of repeats*) $100 /$ chromosome region size. *Number of repeats multiplied by repeat size. For the AA/TT dinucleotides, we used the number of non-overlapped repeats (in Table 3B) multiplied by 4.

\section{Multifractal analysis by group averages}

We also examined the multifractality by group averages of chromosome regions. For all grouped $\mathrm{L}$ and $\mathrm{R}$ regions there is a clear increase in multifractality in comparison with that displayed by all grouped central regions (Figure 4A, Table 4). For all grouped L and R regions, $\tau(\mathrm{q})$ is a nonlinear function, indicating that the nucleotide composition in the C. elegans genome is more aperiodic in the chromosome arms than in the grouped $\mathrm{C}$ regions (Figure 4B).
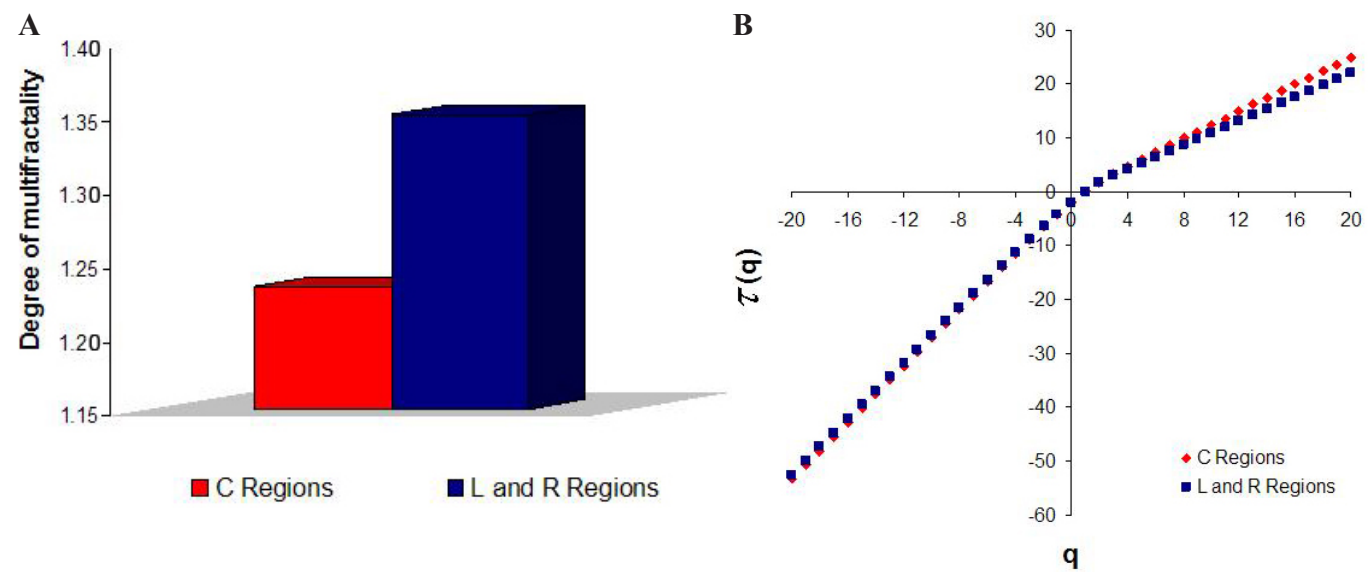

Figure 4. Multifractal analysis by group averages. A. Multifractal degree for averaged groups. B. Multifractal spectra $\tau(q)$ for the two group averages. 


\begin{tabular}{|c|c|c|c|c|c|c|c|}
\hline Chr. region & D-20 & D-2 & D-1 & D1 & D2 & D20 & $\Delta \mathrm{Dq}$ \\
\hline$\overline{\mathrm{IC}}$ & 2.539 & 2.17 & 2.09 & 1.895 & 1.766 & 1.293 & 1.246 \\
\hline II C & 2.534 & 2.168 & 2.088 & 1.902 & 1.786 & 1.331 & 1.203 \\
\hline III C & 2.533 & 2.163 & 2.086 & 1.902 & 1.776 & 1.307 & 1.226 \\
\hline IV C & 2.55 & 2.174 & 2.092 & 1.894 & 1.766 & 1.305 & 1.245 \\
\hline V C & 2.574 & 2.172 & 2.09 & 1.899 & 1.782 & 1.333 & 1.241 \\
\hline $\mathrm{XC}$ & 2.529 & 2.152 & 2.081 & 1.901 & 1.774 & 1.288 & 1.241 \\
\hline Average & & & & & & & 1.234 \\
\hline I L & 2.512 & 2.151 & 2.084 & 1.872 & 1.653 & 1.1 & 1.412 \\
\hline I R & 2.498 & 2.142 & 2.078 & 1.889 & 1.704 & 1.154 & 1.345 \\
\hline II L & 2.503 & 2.138 & 2.074 & 1.898 & 1.728 & 1.186 & 1.317 \\
\hline II R & 2.506 & 2.14 & 2.077 & 1.887 & 1.686 & 1.126 & 1.379 \\
\hline III L & 2.494 & 2.151 & 2.085 & 1.868 & 1.649 & 1.109 & 1.384 \\
\hline III R & 2.505 & 2.151 & 2.084 & 1.873 & 1.66 & 1.109 & 1.397 \\
\hline IV L & 2.516 & 2.144 & 2.08 & 1.882 & 1.688 & 1.142 & 1.374 \\
\hline IV R & 2.533 & 2.165 & 2.092 & 1.87 & 1.684 & 1.162 & 1.371 \\
\hline V L & 2.54 & 2.144 & 2.077 & 1.894 & 1.723 & 1.187 & 1.353 \\
\hline V R & 2.469 & 2.141 & 2.077 & 1.89 & 1.708 & 1.17 & 1.299 \\
\hline X L & 2.513 & 2.139 & 2.075 & 1.898 & 1.742 & 1.199 & 1.314 \\
\hline X R & 2.477 & 2.141 & 2.076 & 1.9 & 1.754 & 1.211 & 1.266 \\
\hline Average & & & & & & & 1.351 \\
\hline
\end{tabular}

\section{DISCUSSION}

We discovered a strong relationship between the multifractal parameters and part of the genetic information coded by the C. elegans genome.

Initially, the nematode's chromosomes were found "poor" in genetic information when compared to the human chromosomes. For instance, chromosome I of C. elegans has a more periodic behavior than chromosome 21 of $H$. sapiens (Figure 1, Panel B1). This is due to numerous regularly arranged elements in the C. elegans genome (Eskesen et al., 2004; Moreno-Herrero, 2006; Spieth and Lawson, 2006; Figure 1, Panels A1, A2). In contrast, human chromosome 21 is richer in variable non-coding sequences ( $98 \%$; Venter et al., 2001$)$ than the C. elegans chromosome ( $74 \%$; Anonymous, 1998$)$.

Subsequently, we found that the nematode's autosomes are more multifractal than the sexual chromosome X, confirming what is known about the molecular structure of the X chromosome, which is particularly poor in genes and repeated DNA sequences (Figure 2, Table 2B; Anonymous, 1998). This difference can also be explained by determining the density by chromosome for genes and repeats from Table 3B ( 79173, 74044, 82492, 79057, 73718, 68618 for chromosomes I, II, III, IV, V, X, respectively). Therefore, we suggest that chromosome $\mathrm{X}$ has a less stable nonlinear structure, which could generate greater genomic instability compared to the autosomes (especially chromosome III).

The nematode's chromosome arms were found to be more multifractal than the central regions of the chromosomes (Figure 3A,B; Table 3A). These results reveal that chromosome arms contain significant correlation structure; these correlations are lower in the central regions of chromosomes. These results also indicate that the observed multifractality is related to nonlinear distributions in the chromosome arms; this is particularly evident in regions where $q>>1$, which are densely populated with nucleotides (Figure 3A), have considerable repetitive DNA. Thus, the repetitive DNA contents located in the $\mathrm{L}$ and $\mathrm{R}$ regions account for the high aperiodicity and genetic variability of these regions. In other words, the multifractal character of these regions can vary greatly due to TTAGGC contents, inverted and tandem 
repeats (Figure 1, Panel A1 and Figure 3C). We only measured 25\% of repetitive element size. This seems to contribute to increasing the degree of multifractality. However, other repetitive DNA elements that were not taken into account might also be involved, as well as other genomic characteristics, for example, high mutation and recombination rates (Wolfe et al., 1989) and $\mathrm{G}+\mathrm{C}$ contents that are mainly observed in the chromosome arms (Anonymous, 1998).

Similarly, group averages are more multifractal in chromosome arms than in central regions. This approach clearly separates the 18 fragments of chromosome regions into two averaged groups; central regions fall into one group and the $\mathrm{L}$ and $\mathrm{R}$ regions into the other (Figure 4A). For all chromosome arms, $\tau(q)$ is a nonlinear function (Figure 4B), indicating that the molecular structure of the chromosome arms has a multifractal behavior. For the central chromosome regions, $\tau(q)$ tends to be closer to linear behavior, especially for $\tau(q \geq 4)$, indicating partial loss of multifractality (Figure 4B). These results suggest that nucleotide fluctuations are less anti-correlated in central regions than in the chromosome arms. A similar behavior that will discriminate group averages has also been observed in another system (Ivanov et al., 1999). These changes in multifractality are also related to the density of group averages for genes and repeats, as can be calculated from Table 3B ( $\sim 81583$ for $\mathrm{L}$ and $\mathrm{R}$ regions and $\sim 65386$ for $\mathrm{C}$ regions).

Finally, we propose a nonlinear model for the structure of the C. elegans genome, with some biological implications. From a multifractal genomics perspective, the detection of a multifractal scaling in the C. elegans genome is of interest because it indicates that the chromosome molecular structure might be organized as a system operating far from equilibrium (Meneveau and Sreenivasan, 1987; Ivanov et al., 1999). This operation can be discussed from three points of view.

1. When the C. elegans genome is compared to the human chromosome, it seems to be less aperiodic. The somewhat lower multifractality of the C. elegans genome is due to its high structural homogeneity, due to many regular characteristics. Lowered non-linearity has been observed in many vital systems close to equilibrium (Goldberger, 1996; Burgos and MorenoTovar, 1996). For example, in the dynamics of the human heartbeat, the loss of multifractality is related to heart failure (Ivanov et al., 1999). That is, the system is operating dangerously close to the equilibrium. In consequence, the lifespan can be dramatically reduced. Thus, we suggest that the $C$. elegans genome might be operating closer to equilibrium than the human genome in order to maintain a simple life cycle.

2. When the C. elegans chromosomes are compared among themselves, less multifractality is observed for the $\mathrm{X}$ chromosome. That means the $\mathrm{X}$ chromosome is operating near equilibrium, which can be translated as major genetic instability. In C. elegans, sex is determined by the opposing actions of $X$ and autosomal signaling elements (Gladden et al., 2007). Additionally, correct chromosome segregation depends upon three coupled processes during meiosis: chromosome pairing, synapses, and recombination (Garcia-Muse and Boulton, 2007; Zetka, 2009). Therefore, the instability of $X$ might contribute to the molecular mechanism of sex determination during meiosis in a selective way (XX or $\mathrm{X} 0$ ). Thus, the $\mathrm{X}$ chromosome would be operating close to equilibrium in order to maintain its particular sexual dimorphism.

3 . When the C. elegans genome is compared by chromosome regions, changes in multifractality are found that are related to the regional organization (in center and arms) exhibited by the chromosome. These behaviors are associated with changes in the contents of repetitive DNA. These results indicate that the chromosome arms are even more complex than previously suspected; since it is there that we found nonlinear properties that take the chromosome away from equilibrium. For example, part of the machinery that regulates several 
physiological processes related to cell proliferation, genome stability, and aging are located in the telomeric regions (Im and Lee, 2005; Cheung and Deng, 2008) in the chromosome arms. Thus, the TTAGGC telomeric sequences would be operating far from equilibrium (perhaps as an attractor of mutations) to protect the genetic information coded by the entire chromosome.

All these biological arguments might explain why the C. elegans genome is organized in a nonlinear manner.

\section{CONCLUSIONS}

We report evidence for multifractality in the C. elegans genome. We demonstrated that this genome has a more periodic behavior than the human genome. We also found changes in multifractality based on other approaches. These changes in multifractality depend upon changes in contents and densities in repetitive DNA and genes. Based on these characteristics, a nonlinear model for the structure of the $C$. elegans genome is proposed. These findings shed light for understanding and quantifying the nonlinear structure organization of the C. elegans genome, which can be extended to other genomes, including the human genome.

\section{ACKNOWLEDGMENTS}

We gratefully acknowledge the Departamento Administrativo de Ciencia, Tecnología e Innovación - COLCIENCIAS of the Republic of Colombia, for supporting this project with Biotechnology Research Grant (\#1103-12-16765). Thanks to Dr. Felipe Garcia for his comments and suggestions. Finally, thanks to the VRI of the University of Cauca for the publication financial support. The authors declare that they have no competing interests.

\section{REFERENCES}

Anonymous (1998). Genome sequence of the nematode C. elegans: a platform for investigating biology. Science 282: 2012-2018.

Berthelsen CL, Glazier JA and Raghavachari S (1994). Effective multifractal spectrum of a random walk. Phys. Rev. E Stat. Phys. Plasmas Fluids Relat. Interdiscip. Topics 49: 1860-1864.

Brenner S and Miller JH (Editors) (2002). Encyclopedia of Genetics. Introduction. Academics Presss, California.

Burgos JD and Moreno-Tovar P (1996). Zipf-scaling behavior in the immune system. Biosystems 39: 227-232.

Cheung AL and Deng W (2008). Telomere dysfunction, genome instability and cancer. Front Biosci. 13: 2075-2090.

Dewey TG and Strait BJ (1996). Multifractals, encoded walks and the ergodicity of protein sequences. Pac. Symp. Biocomput. 216-229.

Eskesen ST, Eskesen FN, Kinghorn B and Ruvinsky A (2004). Periodicity of DNA in exons. BMC Mol. Biol. 5: 12.

$\mathrm{Fu}$ W, Wang Y and Lu D (2007). Multifractal analysis of genomes sequences' CGR graph. Sheng Wu Yi. Xue. Gong. Cheng Xue. Za Zhi. 24: 522-525.

Garcia-Muse T and Boulton SJ (2007). Meiotic recombination in Caenorhabditis elegans. Chromosome Res. 15: 607-621. Gladden JM, Farboud B and Meyer BJ (2007). Revisiting the X:A signal that specifies Caenorhabditis elegans sexual fate. Genetics 177: 1639-1654.

Glazier JA, Raghavachari S, Berthelsen CL and Skolnick MH (1995). Reconstructing phylogeny from the multifractal spectrum of mitochondrial DNA. Phys. Rev. E Stat. Phys. Plasmas Fluids Relat. Interdiscip. Topics 51: 2665-2668.

Goldberger AL (1996). Non-linear dynamics for clinicians: chaos theory, fractals, and complexity at the bedside. Lancet 347: 1312-1314.

Gutiérrez JM, Iglesias A, Rodríguez MA, Burgos JD, et al. (1998). Analyzing the Multifractal Structure of DNA Nucleotide Sequences. In: Chaos and Noise in Biology and Medicine. World Scientific Publishing Co., London, 315-319.

Gutiérrez JM, Rodríguez MA and Abramson G (2001). Multifractal analysis of DNA sequences using a novel chaos-game 
representation. Phys. Stat. Mech. Appl. 300: 271-284.

Halsey TC, Jensen MH, Kadanoff LP, Procaccia I, et al. (1986). Fractal measures and their singularities: The characterization of strange sets. Phys. Rev. A 33: 1141-1151.

Hentschel HGE and Procaccia I (1983). The infinite number of generalized dimensions of fractals and strange attractors. Phys. Nonlinear Phenom. 8: 435-444.

Im SH and Lee J (2005). PLP-1 binds nematode double-stranded telomeric DNA. Mol. Cells 20: 297-302.

Ivanov PC, Amaral LA, Goldberger AL, Havlin S, et al. (1999). Multifractality in human heartbeat dynamics. Nature 399: 461-465.

Jeffrey HJ (1990). Chaos game representation of gene structure. Nucleic Acids Res. 18: 2163-2170.

Lewin B (2008). Genes IX. Jones and Bartlett Publishers Inc., Missinauga.

Mandelbrot B (1982). La Geometría Fractal de la Naturaleza. Tusquets editores, Barcelona.

Meneveau C and Sreenivasan KR (1987). Simple multifractal cascade model for fully developed turbulence. Phys. Rev. Lett. 59: 1424-1427.

Moreno PA, Burgos JD, Vélez PE, Gutiérrez JM, et al. (2000). Multifractal Analysis of Complete Genomes. In: Proceedings of the 12th International Genome Sequencing and Analysis Conference, September 12-15, Miami Beach, 80-81.

Moreno-Herrero F, Seidel R, Johnson SM, Fire A, et al. (2006). Structural analysis of hyperperiodic DNA from Caenorhabditis elegans. Nucleic Acids Res. 34: 3057-3066.

Peitgen HO, Jürgen H and Saupe D (1992). Chaos and Fractals. New Frontiers of Science. Springer-Verlag, New York.

Shannon CE (1948). A mathematical theory of communication. Bell Syst. Tech. J. 27: 379-423, 623-656.

Spieth J and Lawson D (2006). Overview of gene structure. WormBook 18: 1-10.

Stanley HE and Meakin P (1988). Multifractal phenomena in physics and chemistry. Nature 335: 405-409.

Tél T, Fülöp A and Vicsek T (1989). Determination of fractal dimensions for geometrical multifractals. Phys. Stat. Theor. Phys. 159: 155-166.

Venter JC, Adams MD, Myers EW, Li PW, et al. (2001). The sequence of the human genome. Science 291: 1304-1351.

Voss RF (1992). Evolution of long-range fractal correlations and 1/f noise in DNA base sequences. Phys. Rev. Lett. 68 : 3805-3808.

Wolfe KH, Sharp PM and Li WH (1989). Mutation rates differ among regions of the mammalian genome. Nature 337: 283-285.

Wood WB (1987). Introduction to C. elegans Biology. In: The Nematode Caenorhabditis elegans (Wood W, ed.). Cold Spring Harbor Laboratory Press, New York, 1-6.

Yang JY, Zhou Y, Yu ZG, Anh V, et al. (2008). Human Pol II promoter recognition based on primary sequences and free energy of dinucleotides. BMC Bioinformatics 9: 113.

Yu ZG, Anh V and Lau KS (2001). Measure representation and multifractal analysis of complete genomes. Phys. Rev. E Stat. Nonlin. Soft. Matter Phys. 64: 031903.

Yu ZG, Anh V and Lau KS (2003). Multifractal and correlation analyses of protein sequences from complete genomes. Phys. Rev. E Stat. Nonlin. Soft. Matter Phys. 68: 021913.

Yu ZG, Anh V and Lau KS (2004). Chaos game representation of protein sequences based on the detailed HP model and their multifractal and correlation analyses. J. Theor. Biol. 226: 341-348.

Yu ZG, Anh VV, Lau KS and Zhou LQ (2006). Clustering of protein structures using hydrophobic free energy and solvent accessibility of proteins. Phys. Rev. E Stat. Nonlin. Soft. Matter Phys. 73: 031920.

Zetka M (2009). Homologue pairing, recombination and segregation in Caenorhabditis elegans. Genome Dyn. 5: 43-55.

Zhou LQ, Yu ZG, Deng JQ, Anh V, et al. (2005). A fractal method to distinguish coding and non-coding sequences in a complete genome based on a number sequence representation. J. Theor. Biol. 232: 559-567.

\section{Description of additional data files}

\section{Additional file 1. Multifractal and molecular information.}

This file has the information for all multifractal and molecular parameters calculated for our study. 


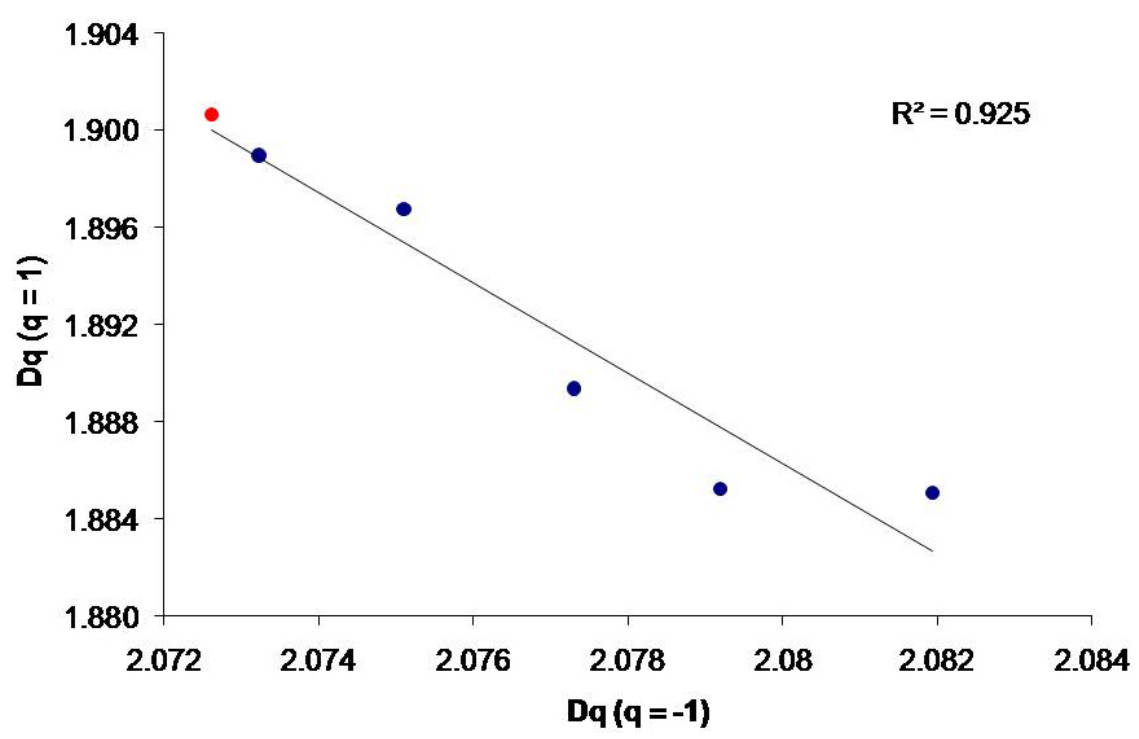

Additional Figure 2C. Distribution of 2-D points (D-1, D1) of the Caenorhabditis elegans chromosomes.

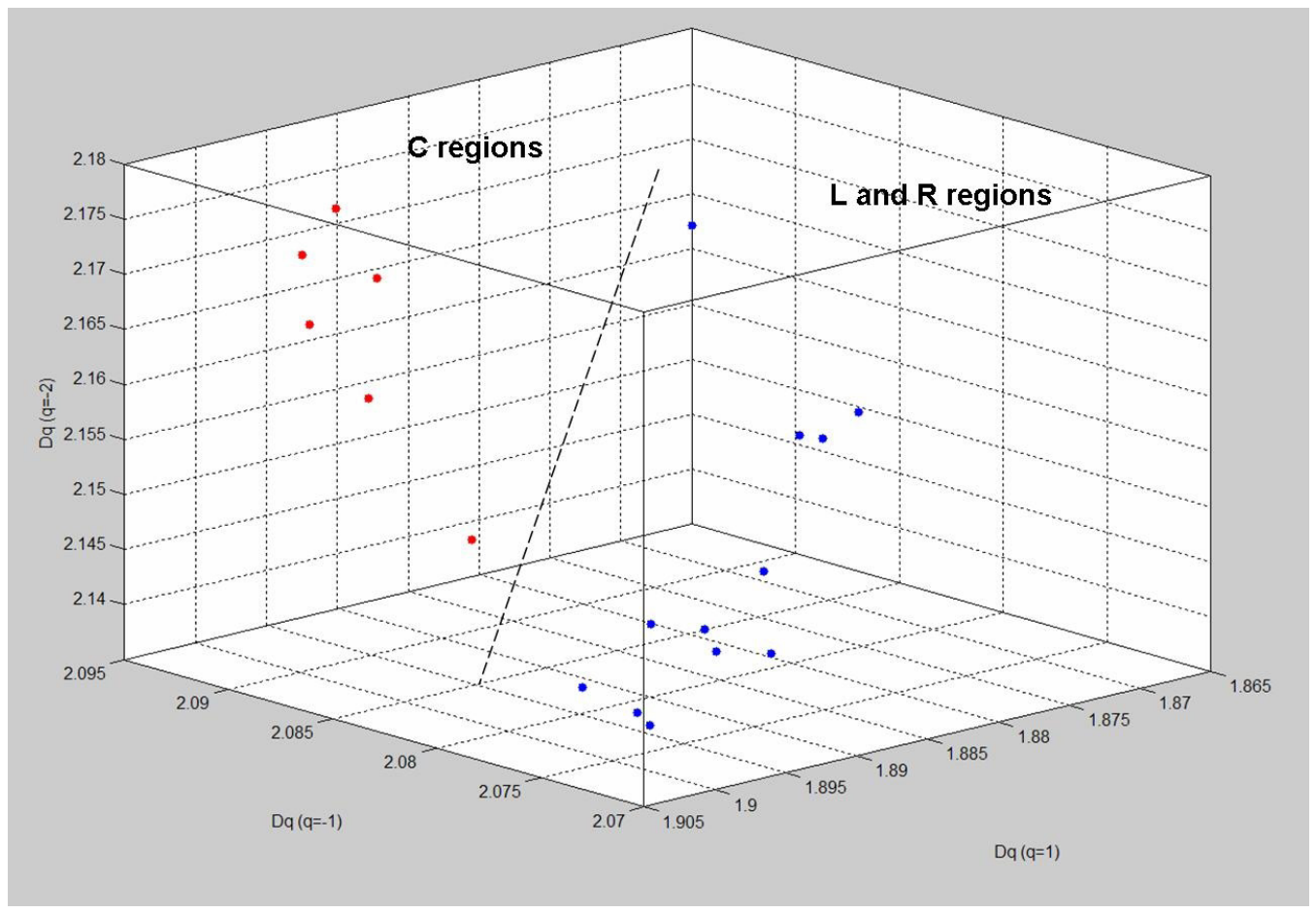

Additional Figure 3E. Multifractality by chromosome region. Distribution of 3-D points (D-1, D1, D-2) of the Caenorhabditis elegans chromosome fragments. 